\title{
A Novel Shape Generation Method Applied in Product Design Yan Zhou ${ }^{1, a}$, Chenxiao Fan ${ }^{2, b^{*}}$ \\ ${ }^{1}$ School of Mechatronics Engineering, Harbin Institute of Technology, Harbin, China \\ ${ }^{2}$ School of Mechatronics Engineering, Harbin Institute of Technology, Harbin, China \\ azhouyan@hit.edu.cn, ${ }^{b}$ chenxiaofanfcx@163.com
}

Keywords: Shape extraction, Fusion method, Shape averaging, Spline interpolation Abstract. In the traditional product design method, the design method simply based on the knowledge and experience has hindered the efficiency of the product design, and it brings inconvenience to predict the tendency of the product shape. To solve this problem, a shape generation method is proposed, based on averaging algorithm and spline interpolation. A sampling method for product model based on 2D section is put forward, the coordinates of the sampling points of the 3D model are extracted through setting up the 2D sections. The classification of the 3D model in the semantic level is determined. After the shape extraction, the G2 organic spline curve is generated based on shape averaging method and spline interpolation, thus providing basis for the new shape generation. The shape fusion of the designing mouse is taken as an example to prove the feasibility and validity of this approach. It turned out that the approach proposed in the paper can generate organic surface, which can effectively preserve the source shape. This method proves to be useful for predicting the development trend of product shape or extracting feature templates from a set of related shapes.

\section{Introduction}

In the generation process of a new shape, the shape extraction, denoising and fusion are 3 key parts, which are based on the computer animation, surface reconstruction technology and research of computational mathematics. In the traditional industrial design method, the perceptual design method often bring inconvenience for the projects. Aesthetics should be like other functional levels in design by quantitative analysis ${ }^{[1]}$. Shenchang Eric Chen et al. presented the computer aided shape averaging method $^{[2]}$. But it didn't consider the average in the semantic level. For a shape of higher complexity.it can not distinct in the semantic level.

This paper proceeds as follow to solve the problems of the shape extraction and fusion deformation in the computer $3 \mathrm{~d}$ model. In the extraction phase, allowing the classification in the semantic level for the extracted model. Then the source shape point sampling is completed, and the new shape is generated using weighted averaging and spline interpolation. In the $2 \mathrm{~d}$ graphics interpolation, the quadratic spline function is used to generate a G2 continuous interpolation curve. This method is proposed based on the design of the mouse appearance.

\section{Shape extraction method}

The first step is to convert an ordinary $3 \mathrm{~d}$ mouse model into the source shape. The $3 \mathrm{~d}$ mouse model in this research is got by direct modeling method. The mouse appearance on the semantic structure is divided into four parts. Namely, button, pulley, auxiliary holding structure and base. The body and buttons of the two source shapes are fused respectively,which is shown in Fig.1.

The more sampling points there are, the more intensive the model is, but it may bring the disadvantage of the computing redundancy. so the selection of sampling points is required to ensure computing efficiency model and can effectively keep the key source of surface structure information. Application of the $2 \mathrm{~d}$ slices can effectively keep the coordinate information of the key points, which provides convenience for the standardized processing of the sampling points information. The slicing process is shown in Fig.2. 


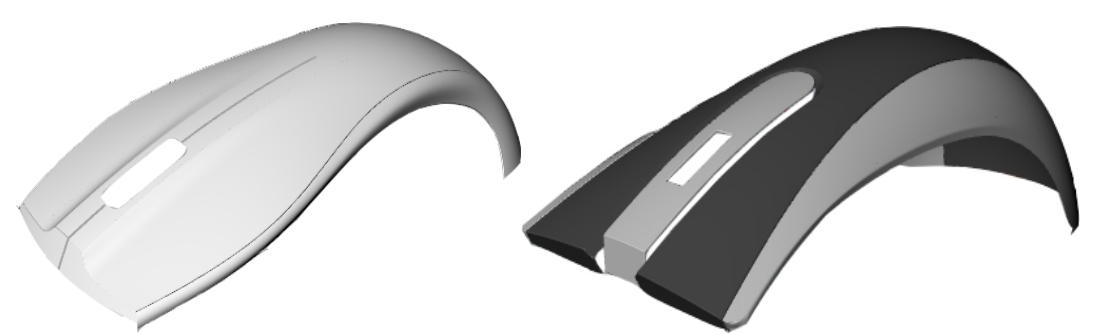

Fig.1. Two source mouse surface shape after processing
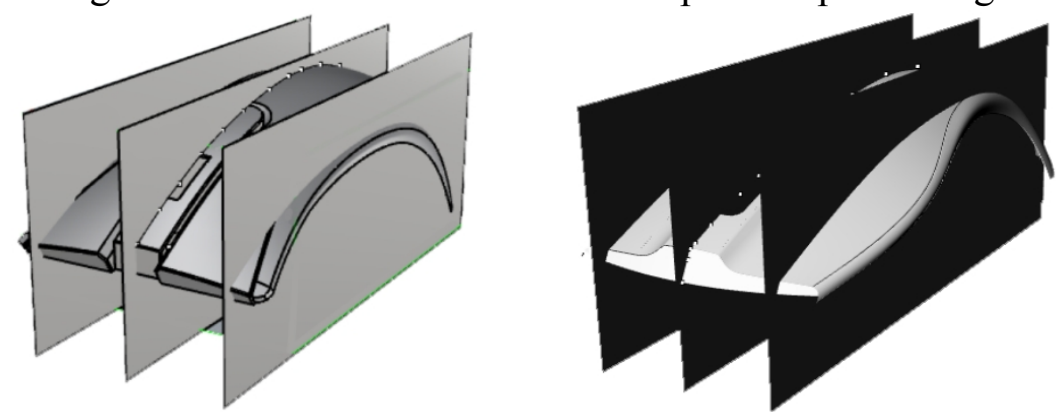

Fig.2. $2 \mathrm{~d}$ slicing treatment of the input shapes

After $2 \mathrm{~d}$ slicing treatment, the sampling points of each slice are selected,which requires to preserve the main characteristics of the input shape. It should also avoid causing computational difficulties. As is shown in Fig.3 is the sampling points in one slice. Sampling points at region A are more intense, locally strengthened the restrictions on the shape outline. This is suitable in a sharp corner position. Spacing of sampling points in B is larger, thus reduced the amount of calculation of the next step.

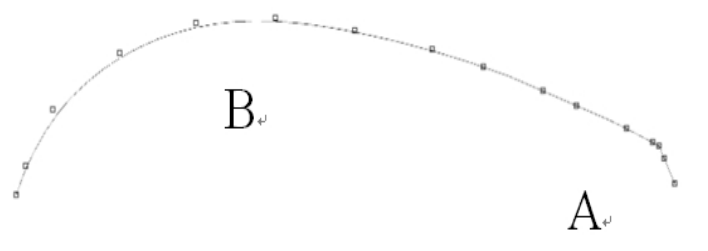

Fig.3. $2 \mathrm{~d}$ slicing treatment of the input shapes

In the sampling points generating process, the source shape has been distinguished from the semantic level, the metaphorical factors in the semantic level have been taken into account.

\section{Shape construction method}

This section describes setting up the correspondence of the sampling points from the source shape.This weighted averaging method is used to get the new coordinates. Quadratic spline interpolation is used to get the G2 continuous sampling contour, which is finally utilized to develop a new shape.

The points number has been set the same in the point generating process,so we just need to choose the starting point,as is shown in Fig.4.There are 22 pairs of corresponding points in this figure. $1 \mathrm{P}$ and 1Q from source shape 1 and 2 are the starting point respectively. We made a corresponding attachment from them.

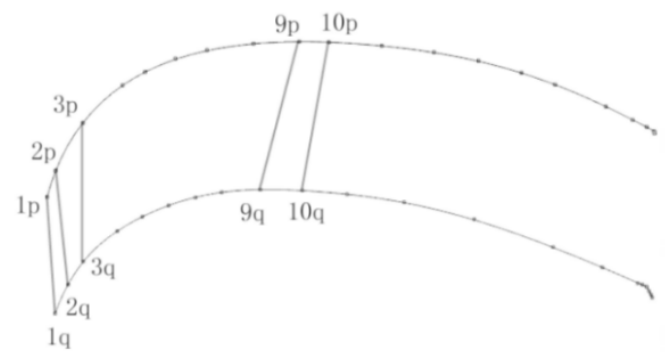

Fig.4. Slice sampling points corresponding process 
Shape can be averaged in both semantic level or grammatical level. We define the two groups of input contour curve A (top) and curve B (below) in Fig.4 respectively, the new curve generated is defined as C, key point coordinates on the curve C should be got. Sampling points set on Curve of A and $\mathrm{B}$ are:

$$
\begin{aligned}
& S_{A}=\left\{\alpha_{p}, 1 \leq p \leq 22\right\} \\
& S_{B}=\left\{\beta_{q}, 1 \leq q \leq 22\right\}
\end{aligned}
$$

Then this shape averaging method can be defined as:

$$
\mathrm{S}_{\mathrm{C}}=\mathrm{S}_{\mathrm{A}} \quad \mathrm{S}_{\mathrm{B}}=\left\{\mathrm{a}_{\mathrm{p}} \quad \beta_{\mathrm{q}}, 1 \leq \mathrm{p} \leq 22,1 \leq \mathrm{q} \leq 22\right\}
$$

Symbol ' ' represents a weighted averaging deformation operator, define it as:

$$
\alpha_{\mathrm{p}} \quad \beta_{\mathrm{q}}=(1-\lambda) \alpha_{\mathrm{p}}+\lambda \beta_{\mathrm{q}}, \lambda \in \infty
$$

$\mathrm{SA}, \mathrm{SB}$ and SC are the sampling point sets on curve A,B and $\mathrm{C}$ respectively, $\lambda$ is the weighted averaging coefficient in the deformation process, which takes an infinite in real number. The value of $\lambda$ determines which sampling curve's feature will be preserved more by the curve $C$. We set $\lambda=0.5$ for this case. There are 22 key points on curve $C$, which are the middle points between the sampling points on input curve A and B in geometry, as is shown in Fig.5.

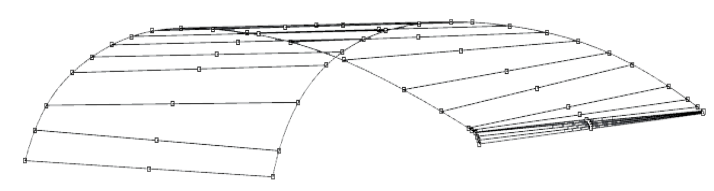

Fig.5. Key points positions on curve $\mathrm{C}$

The 22 points are utilized as the interpolation points on the generated new curve with interpolation method. K.C.HUI used the sharp corner to split the curve, establishing the segment correspondence according to the segment similarity criterion, then used linear interpolation to set up each sampling points uniformly distributed on each segment ${ }^{[3]}$. So we can get a smooth interpolation curve over a series of sampling points using the quadratic spline interpolation method. This curve can remain $\mathrm{G}_{2}$ continuity. Do straight L through points M1, M2, the linear algebraic equation of L1, L2, L is:

$$
\left\{\begin{array}{l}
\mathrm{L}_{1}(x, y)=\mathrm{a}_{1} x+\mathrm{b}_{1} y+\mathrm{c}_{1}=0 \\
\mathrm{~L}_{2}(x, y)=\mathrm{a}_{2} x+\mathrm{b}_{2} y+\mathrm{c}_{2}=0 \\
\mathrm{~L}(x, y)=\mathrm{a} x+\mathrm{b} y+\mathrm{c}=0
\end{array}\right.
$$

Do curve C:

$$
\mathrm{f}(x, y)=\mathrm{L}_{1}(x, y) \mathrm{L}_{2}(x, y)+\mathrm{KL}^{\mathrm{n}}(x, y)=0
$$

$\mathrm{n}$ is an integer greater than 1, equation (5) indicates that the curve segment between M1 and M2 of curve $\mathrm{C}$ is the splines. If the curvature at point $\mathrm{C}$ is known as $\Theta$. According to the theory put forward by Sanyuan zhang ${ }^{[4]}, \mathrm{n}=2$, the parameter $\mathrm{K}$ can be expressed as:

$$
K=\frac{\Theta\left(\mathrm{a}_{2} x_{1}+b_{2} y_{1}\right)\left(a_{1}^{2}+b_{1}^{2}\right)^{3}}{2\left(a_{1} b-a b_{1}\right)^{2}}
$$


Use straight lines to connect the key points on curve C, as is shown in Fig 6 (a). As the tangent at $\mathrm{P} 1$ has been given, we utilize it to determine the tangents at P2,P3,..,Pn. The polygonal line in Fig.6 (b) is a portion from Fig 6 (a).

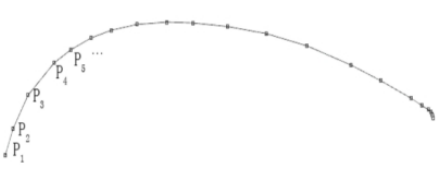

(a)

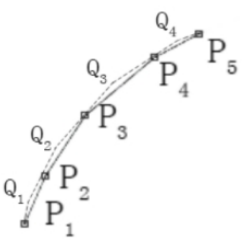

(b)

Fig.6. Connecting the key points using the straight lines

Do linear line Q1P2Q2 over P2 intersecting with tangent P1Q1 over P1 at Q1, then in order do a straight line $\mathrm{Q} i-1 \mathrm{P} i \mathrm{Q} i$ over $\mathrm{P} i$ intersecting with linear line $\mathrm{Q} i-2 \mathrm{P} i-1 \mathrm{Q} i-1$ at $\mathrm{Q} i-1,(i=3, \ldots, \mathrm{n})$, determination of the Qn-1 is to ensure that the triangle Qn Pn-1 Pn is an isosceles triangle( $\mathrm{n}=22$ in this case ). After determining the tangents, we construct the quadratic curve section $\mathrm{C}_{1}$ over $\mathrm{P} 1$ and $\mathrm{P} 2$ with the G2 continuity curve using tangent P1Q1, Q1P2 and K. In the same way the complete quadratic interpolation spline is determined, which is shown in Fig.7 (a). This is a 2d interpolation spline with 22 key points P1, P2,..,P22. After the completion of the above work, we can easily use the 3D modeling software to generate a new organic mouse surface, as shown in Fig.7 (b) and Fig.7 (c).

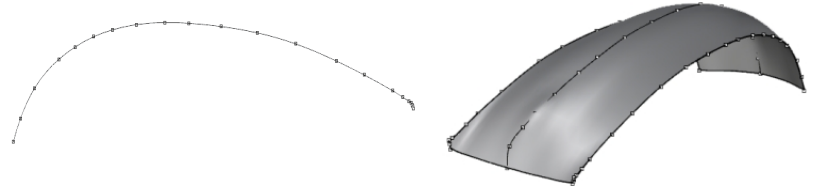

(a)

(b)

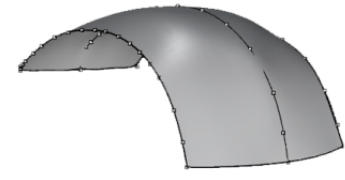

(c)

Fig.7. A mouse contour generated by the quadratic interpolation spline(a) ; the new main body shape of the mouse model based on 3 spline curves $(b, c)$

\section{Conclusions}

This paper proposed a shape extraction and shape fusion method based on 3D modeling software, and a new contour curve is generated by using the spline interpolation method. First, we used the 2D slices to split the input shape to get the information of it. The shape fusion is achieved by the weighted averaging method. The quadratic spline interpolation is introduced to generate organic curves with G2 continuity using key points, and finally the organic surfaces are output by the computer according to the 3 fusion curves. According to the method provided by this paper, designers can quickly get the product shape to meet the needs of customers. Using the method above, the designer can also predict the trend of product shape according to the evolution history of the products.

\section{Acknowledgement}

This study was completed in the Department of Industrial Design of Harbin Institute of Technology, many thanks to Yan Zhou for the help provided for this study.

\section{References}

[1] D. Coates, "Understanding Aesthetics: From Old Shoes to a Teacup," Industrial Design, Sept./Oct. 1979, pp. 32-35.

[2] S. Chen. A Computer Assisted Approach to Shape Averaging and Its Applications to Industrial Design, master's thesis, Ohio State Univ., 1986. 
[3] K.C.Hui, Li Y. a feature based shape blending technique for industrial design[J],CAD, 1998,30(10);828-834.

[4] Sanyuan Zhang . Interpolating 2D Data Set by Conic Spline with G2 Continuity [J]. Journal of computer aided design and computer graphics, 2000, 12 (6). DOI:10.3321/j.issn:1003-9775.2000.06.005.(In Chinese). 